Research Article

\title{
Approximate Calculation Method for Noncentral t-Distribution Quantile
}

\author{
Jun Gao $\mathbb{D i D}^{1,2}$ and Jitao Yao $\mathbb{i D}^{1,2}$ \\ ${ }^{1}$ College of Civil Engineering, Xi'an University of Architecture and Technology, Xi'an 710055, China \\ ${ }^{2}$ Key Lab of Structural Engineering and Earthquake Resistance, Ministry of Education (XAUA T), Xi'an 710055, China
}

Correspondence should be addressed to Jitao Yao; yaojitao1224@163.com

Received 13 March 2021; Accepted 7 May 2021; Published 25 May 2021

Academic Editor: Emilio Gómez-Déniz

Copyright (c) 2021 Jun Gao and Jitao Yao. This is an open access article distributed under the Creative Commons Attribution License, which permits unrestricted use, distribution, and reproduction in any medium, provided the original work is properly cited.

\begin{abstract}
In the process of structural design and structural performance evaluation, the inference of the reliable life of the structure and the representative value of the material strength is a necessary work. The determination of material strength is the presumption of the quantile of the normal distribution, and the determination of the confidence level of the quantile of the normal distribution involves the noncentral t-distribution function. However, the calculation of the quantile is very complicated and is often provided in the form of a numerical table, which often involves multiparameter interpolation calculation, so it is not convenient to apply. The existing approximate calculation methods for noncentral $t$-distribution quantiles have strict application conditions, and the calculation process is relatively cumbersome. It is still difficult to meet actual needs in terms of fitting accuracy, application range, and convenience. In this paper, a new calculation method for noncentral t-distribution quantiles is proposed by introducing new probability expressions and related approximate distributions, based on theoretical derivation and numerical fitting. The comparative analysis results show that the method not only is convenient for calculation but also has the advantages of higher accuracy and wider application range, and it is more in line with the actual needs of engineering.
\end{abstract}

\section{Introduction}

The structural reliability design covers the entire civil engineering and is the basic guarantee for the normal operation of the building structures $[1,2]$. There are various reliability modeling methods. In practical engineering, uncertain failure modes can result in the system failure [3-5]. The noncentral $\mathrm{t}$-distribution is mainly used for small sample inference of normal distribution quantile, and it is widely used in the estimation of structural reliability life, representative value of material strength, and so on $[6,7]$. The probability density function of the noncentral t-distribution is complex, and its quantile is usually calculated by numerical methods and provided in the form of a numerical table. However, in the current numerical table, only some typical values are provided, and in other cases, interpolation calculation is required, and multiple parameters are involved, which is inconvenient to apply [8-11]. Therefore, a variety of approximate calculation methods have been proposed, but they are still difficult to meet the actual needs in terms of fitting accuracy, scope of application, and convenience $[12,13]$.

In this paper, a new calculation method for noncentral $\mathrm{t}$-distribution quantiles is proposed by introducing new probability expressions and related approximate distributions, based on theoretical derivation and numerical fitting. The comparative analysis results show that the method not only is convenient for calculation, but also has the advantages of higher accuracy and wider application range, and it is more in line with the actual needs of engineering.

\section{Current Approximate Calculation Method}

The random variable $X$ obeys the normal distribution $N(\mu$, $\sigma^{2}$ ), and the distribution parameters $\mu$ and $\sigma^{2}$ are unknown. Then, the upper $p$ and $1-p$ quantile values of $X$ can be expressed as 


$$
\begin{aligned}
x_{p} & =\mu-z_{1-p} \sigma, \\
x_{1-p} & =\mu+z_{1-p} \sigma,
\end{aligned}
$$

where $z_{1-p}$ is the upper $1-p$ quantile of the standard normal distribution; $p$ is the assurance rate of $X \geq x_{p}$ or $X \leq x_{1-p}$, and its value is generally more than 0.5 , and $x_{p}$ and $x_{1-p}$ are small and large quantile values, respectively.

Let $X_{1}, X_{2}, \ldots, X_{n}$ be the $n$ samples of the random variable $X$. According to the statistical method, after obtaining the sample realization value $x_{1}, x_{2}, \ldots, x_{n}$, the lower limit estimate of $x_{p}$ and the upper limit estimate of $x_{1-p}$ are, respectively,

$$
\begin{aligned}
\hat{x}_{p} & =\bar{x}-k s, \\
\hat{x}_{1-p} & =\bar{x}+k s,
\end{aligned}
$$

where

$$
\begin{aligned}
& \bar{x}=\frac{1}{n} \sum_{i=1}^{n} x_{i}, \\
& s=\sqrt{\frac{1}{n-1} \sum_{i=1}^{n}\left(x_{i}-\bar{x}\right)^{2},} \\
& k=\frac{t\left(n-1, z_{1-p} \sqrt{n}, \alpha\right)}{\sqrt{n}},
\end{aligned}
$$

where $t_{\left(n-1, z_{1-p} \sqrt{n}, \alpha\right)}$ is the upper $\alpha$ quantile value of the noncentral $t$-distribution with a degree of freedom of $X$ and a parameter of $z_{1-p} \sqrt{n}$, and $\alpha$ is the significance level.

The coefficient $k$ is related to the sample size $n$, the distribution parameter $z_{1-p} \sqrt{n}$ and the significance level $\alpha$, etc., and is mainly determined by the quantile value $t_{\left(n-1, z_{1-p} \sqrt{n}, \alpha\right)}$ of the noncentral t-distribution. According to the approximate statistics of the $F$ distribution [12], it is recommended to approximate as

$$
t_{(n-1, \lambda, \alpha)}= \begin{cases}\frac{\lambda}{C_{n}} F_{\left(\gamma_{1}, \gamma_{1}, \alpha\right)}, & \lambda>0, \\ \frac{\lambda}{C_{n}} F_{\left(\gamma_{1}, \gamma_{1}, 1-\alpha\right)}, & \lambda<0,\end{cases}
$$

where

$$
\begin{aligned}
& C_{n}=\sqrt{\frac{2}{n-1}} \frac{\Gamma(n / 2)}{\Gamma((n-1) / 2)}, \\
& \gamma_{1}=2 \lambda^{2}, \\
& \gamma_{2}=\frac{2 C_{n}^{2}}{1-C_{n}^{2}},
\end{aligned}
$$

where $t_{(n-1, \gamma, \alpha)}$ is the upper $\alpha$ quantile value of the noncentral $\mathrm{t}$-distribution with the degree of freedom $n-1$ and the parameter $\gamma \cdot F_{\left(\gamma_{1}, \gamma_{1}, \alpha\right)}$ and $F_{\left(\gamma_{1}, \gamma_{1}, 1-\alpha\right)}$ are the upper $\alpha$ and $1-\alpha$ quantile values of the $F$ distribution with parameters $\gamma_{1}$ and $\gamma_{2}$, respectively. When $\gamma_{1}$ and $\gamma_{2}$ are nonpositive integers, the values of $F_{\left(\gamma_{1}, \gamma_{1}, \alpha\right)}$ and $F_{\left(\gamma_{1}, \gamma_{1}, 1-\alpha\right)}$ can be determined by linear interpolation method. This method is mainly applicable to the case of $n>9$, and, in general, it is still inconvenient to check the table.

When

$$
\begin{aligned}
T_{1} & =\frac{\bar{X}-x_{p}}{S / \sqrt{n}}, \\
\bar{X} & =\frac{1}{n} \sum_{i=1}^{n} X_{i}, \\
S & =\sqrt{\frac{1}{n-1} \sum_{i=1}^{n}\left(X_{i}-\bar{X}\right)^{2} .}
\end{aligned}
$$

Then, $T_{1}$ obeys a noncentral $\mathrm{t}$-distribution with a degree of freedom of $n-1$ and a parameter of $z_{1-p} \sqrt{n}$. Jitao [14] approximates that the probability distribution of $S$ is a normal distribution $N\left(\sigma,\left(\sigma^{2} / 2(n-1)\right)\right)$. By numerical fitting, it is recommended to approximate the noncentral $\mathrm{t}$-distribution of the quantile value as

$$
\begin{aligned}
t_{(n-1, \gamma, \alpha)} & =\gamma+z_{\alpha} \sqrt{1-\left(\frac{\left.\left(z_{\alpha}^{2}-\gamma^{2}\right) /(2(n-m))\right)}{1-\left(z_{\alpha}^{2} /(2(n-m))\right) z_{\alpha}^{2} \leq 2(n-m)},\right.} \\
m & =\frac{1.5}{(1-\alpha)^{2}}
\end{aligned}
$$

where $z_{\alpha}$ is the upper $\alpha$ quantile value of the standard normal distribution.

This method is mainly applicable to the case of $n \geq 5$, has a wider scope of application, and is simpler than the method proposed by Huirong [12]. But it can be known from the comparison analysis of the accuracy of the following, the approximation methods proposed by Huirong [12] and Jitao and Yaokui [13] are difficult to meet the actual needs in terms of accuracy, and the scope of application is relatively limited.

\section{New Approximate Calculation Method}

If

$$
T_{2}=\frac{U+\gamma}{\sqrt{V /(n-1)}},
$$

where $U$ obeys the standard normal distribution and $V$ obeys the chi-square distribution with degrees of freedom of $n-1$, then, $T_{2}$ obeys a noncentral $t$-distribution with a degree of freedom of $n-1$ and a parameter of $\gamma$. When 


$$
\begin{aligned}
\mathrm{P}\left\{T_{2} \leq t_{(n-1, \gamma, \alpha)}\right\} & =\mathrm{P}\left\{\frac{U+\gamma}{\sqrt{V /(n-1)}} \leq t_{(n-1, \gamma, \alpha)}\right\} \\
& =1-\alpha \\
W & =\sqrt{\frac{V}{n-1}} .
\end{aligned}
$$

Then,

$$
P\left\{U+\gamma-t_{(n-1, \gamma, \alpha)} W \leq 0\right\}=1-\alpha
$$

The probability density function and mean value of $V$ are

$$
\begin{aligned}
f_{V}(v) & =\frac{1}{2^{(n-1) / 2} \Gamma((n-1) / 2)} e^{-(v / 2)} v^{((n-1) / 2)-1}, \quad v \geq 0, \\
\mu_{V} & =n-1 .
\end{aligned}
$$

So, the probability density function of $W$ is

$$
f_{W}(w)=2(n-1) w f_{V}\left[(n-1) w^{2}\right], \quad w \geq 0 .
$$

Therefore,

$$
\begin{aligned}
\mu_{W} & =\int_{0}^{\infty} w f_{W}(w) \mathrm{d} w \\
& =\int_{0}^{\infty} \sqrt{\frac{v}{n-1}} f_{V}(v) \mathrm{d} v \\
& =\sqrt{\frac{2}{n-1}} \frac{\Gamma(n / 2)}{\Gamma((n-1) / 2)}, \\
E\left(W^{2}\right) & =\int_{0}^{\infty} w^{2} f_{W}(w) \mathrm{d} w \\
& =\int_{0}^{\infty} \frac{v}{n-1} f_{V}(v) \mathrm{d} v=1, \\
\sigma_{W} & =\sqrt{E\left(W^{2}\right)-\mu_{W}^{2}} \\
& =\sqrt{1-\frac{2}{n-1} \frac{\Gamma^{2}(n / 2)}{\Gamma^{2}((n-1) / 2)}} .
\end{aligned}
$$

Referring to the method proposed by Shisong et al. [15] and Jitao [14], approximating that $W$ obeys the normal distribution $N\left(\mu_{W}, \sigma_{W}^{2}\right)$, then

$$
\begin{aligned}
& \mathrm{P}\left\{U+\gamma-t_{(n-1, \gamma, \alpha)} W \leq 0\right\} \approx \Phi\left(-\frac{\gamma-t_{(n-1, \gamma, \alpha)} \mu_{W}}{\sqrt{1+t_{(n-1, \gamma, \alpha)}^{2} \sigma_{W}^{2}}}\right)=1-\alpha, \\
& \frac{t_{(n-1, \gamma, \alpha)} \mu_{W}-\gamma}{\sqrt{1+t_{(n-1, \gamma, \alpha)}^{2} \sigma_{W}^{2}}} \approx z_{\alpha .}
\end{aligned}
$$

It can be obtained that

$$
\begin{gathered}
t_{(n-1, \gamma, \alpha)}=\frac{\gamma+z_{\alpha} \sqrt{1-\left(z_{\alpha}^{2}-\gamma^{2}\right) \delta_{W}^{2}}}{\mu_{W}\left(1-z_{\alpha}^{2} \delta_{W}^{2}\right)}, \\
\delta_{W}=\frac{\sigma_{W}}{\mu_{W}}=\sqrt{\frac{n-1}{2} \frac{\Gamma^{2}((n-1) / 2)}{\Gamma^{2}(n / 2)}-1} \approx 1-\frac{0.25}{n-1} .
\end{gathered}
$$

After the overall numerical fit, it can be found in equation (16):

$$
\begin{aligned}
\mu_{W} & =1-\frac{0.25}{n-1}\left(p+0.6-\frac{0.1}{\alpha}\right), \\
p & =\Phi\left(\frac{\gamma}{\sqrt{n}}\right) .
\end{aligned}
$$

If

$$
\gamma=z_{1-p} \sqrt{n}
$$

then

$$
k=\frac{z_{1-p} \sqrt{n}+z_{\alpha} \sqrt{1-\left(z_{\alpha}^{2}-z_{1-p}^{2} n\right) \delta_{W}^{2}}}{\mu_{W} \sqrt{n}\left(1-z_{\alpha}^{2} \delta_{W}^{2}\right)} .
$$

\section{Comparative Analysis}

Compare and analyze the calculation accuracy of the four methods: method 1: the method proposed by Huirong [12] calculates $k$ according to (4); method 2: the method proposed by Jitao [13] calculates $k$ according to (7); method 3: the method in this paper calculates $k$ according to (20). Tables 1-5 list the corollary results of the coefficient $k$ according to the different values of $C$.

In order to better research the trend of the coefficient $k$ corollary results, the relative error scatter plots of the coefficient $k$ corollary results can be drawn when $C=0.5$, $C=0.6, C=0.7, C=0.8$, and $C=0.9$, respectively, as shown in Figures 1-5. Since the relative error of Method 2 when $n<5$ is too large, this part of the data is deleted when the figure is made.

From the results of comparative analysis, the error range of method 1 is $-30.59 \% \sim 0.84 \%$, the error range of method 2 is $-28.36 \% \sim 104.4 \%$, and the error range of method 3 is $-3.86 \% \sim 2.75 \%$. Separately, when $p=0.7$, the relative error ranges of these three methods are $-30.59 \% \sim 0.84 \%,-21.6 \%$ $76.29 \%$, and $-3.86 \% \sim 1.67 \%$; when $p=0.8$, the relative error ranges of these three methods are $-9.9 \% \sim 0.24 \%,-28.36 \%$ $91.33 \%$, and $-2.81 \% \sim 2.0 \%$; when $p=0.9$, the relative error ranges of these three methods are $-10.11 \% \sim-0.01 \%$, $-14.5 \sim 101.86 \%$, and $-2.45 \% \sim 2.11 \%$; and when $p=0.95$, the relative error ranges of these three methods are $-10.5 \%$ $0.85 \%,-5.02 \% \sim 104.4 \%$, and $-2.35 \% \sim 2.75 \%$. It can be obtained that the error range calculated by method 2 is the largest, method 1 is in the middle, and method 3 is the smallest, indicating that the relative error calculated by method 3 is the smallest with the highest precision. 
TABLE 1: Corollary results of coefficient $k(C=0.5)$.

\begin{tabular}{|c|c|c|c|c|c|c|c|}
\hline$p$ & & & & 0.7 & & & \\
\hline$n$ & Exact solution & Method 1 & Relative error (\%) & Method 2 & Relative error (\%) & Method 3 & Relative error $(\%)$ \\
\hline 3 & 0.597986 & 0.415034 & -30.59 & 0.524401 & -12.31 & 0.608001 & 1.67 \\
\hline 4 & 0.572487 & 0.435142 & -23.99 & 0.524401 & -8.40 & 0.577322 & 0.84 \\
\hline 5 & 0.560064 & 0.449987 & -19.65 & 0.524401 & -6.37 & 0.563115 & 0.54 \\
\hline 6 & 0.552664 & 0.460839 & -16.61 & - & - & 0.554921 & 0.41 \\
\hline 7 & 0.547827 & 0.469046 & -14.38 & 0.524401 & -4.28 & 0.54959 & 0.32 \\
\hline 8 & 0.544407 & 0.475423 & -12.67 & 0.524401 & -3.68 & 0.545844 & 0.26 \\
\hline 9 & 0.541868 & 0.480519 & -11.32 & 0.524401 & -3.22 & 0.543068 & 0.22 \\
\hline 10 & 0.539877 & 0.484634 & -10.23 & 0.524401 & -2.87 & 0.585111 & 0.19 \\
\hline 20 & 0.53166 & 0.503979 & -5.21 & 0.524401 & -1.37 & 0.562683 & 0.08 \\
\hline 30 & 0.529147 & 0.510691 & -3.49 & 0.524401 & -0.90 & 0.554224 & 0.05 \\
\hline 40 & 0.527907 & 0.514103 & -2.61 & 0.524401 & -0.66 & 0.549534 & 0.04 \\
\hline Error range & & & $-30.59 \sim-2.61$ & & $-12.31 \sim-0.66$ & & $0.04 \sim 1.67$ \\
\hline$p$ & & & & 0.8 & & & \\
\hline$n$ & Exact solution & Method 1 & Relative error (\%) & Method 2 & Relative error (\%) & Method 3 & Relative error (\%) \\
\hline 3 & 0.970715 & 0.88509 & -8.82 & 0.841621 & -13.3 & 0.990143 & 2 \\
\hline 4 & 0.924547 & 0.859236 & -7.06 & 0.841621 & -9.0 & 0.935135 & 1.15 \\
\hline 5 & 0.902606 & 0.849782 & -5.85 & - & - & 0.909861 & 0.8 \\
\hline 6 & 0.889844 & 0.845484 & -4.99 & - & - & 0.895342 & 0.62 \\
\hline 7 & 0.881479 & 0.843315 & -4.33 & 0.841621 & -4.52 & 0.885917 & 0.5 \\
\hline 8 & 0.875564 & 0.842031 & -3.83 & 0.841621 & -3.88 & 0.879306 & 0.43 \\
\hline 9 & 0.871202 & 0.841351 & -3.43 & 0.841621 & -3.4 & 0.874412 & 0.37 \\
\hline 10 & 0.867798 & 0.840878 & -3.1 & 0.841621 & -3.02 & 0.870643 & 0.33 \\
\hline 20 & 0.853853 & 0.840228 & -1.6 & 0.841621 & -1.43 & 0.855123 & 0.15 \\
\hline 30 & 0.849585 & 0.840502 & -1.07 & 0.841621 & -0.94 & 0.850419 & 0.1 \\
\hline 40 & 0.847534 & 0.84068 & -0.81 & 0.841621 & -0.7 & 0.848145 & 0.07 \\
\hline Error range & & & $-8.82 \sim-0.81$ & & $-13.3 \sim-0.7$ & & $0.07 \sim 2$ \\
\hline$p$ & & & & 0.9 & & & \\
\hline$n$ & Exact solution & Method 1 & Relative error (\%) & Method 2 & Relative error (\%) & Method 3 & Relative error (\%) \\
\hline 3 & 1.498545 & 1.481939 & -1.11 & 1.281552 & -14.5 & 1.530211 & 2.11 \\
\hline 4 & 1.418883 & 1.403378 & -1.09 & - & - & 1.437254 & 1.29 \\
\hline 5 & 1.381884 & 1.368009 & -1 & - & - & 1.394886 & 0.94 \\
\hline 6 & 1.360419 & 1.348175 & -0.9 & - & - & 1.370643 & 0.75 \\
\hline 7 & 1.346566 & 1.335561 & -0.82 & 1.281552 & -4.83 & 1.354944 & 0.62 \\
\hline 8 & 1.336729 & 1.326928 & -0.73 & 1.281552 & -4.13 & 1.343949 & 0.54 \\
\hline 9 & 1.32965 & 1.320672 & -0.68 & 1.281552 & -3.62 & 1.335819 & 0.46 \\
\hline 10 & 1.324126 & 1.315862 & -0.62 & 1.281552 & -3.22 & 1.329564 & 0.41 \\
\hline 20 & 1.301272 & 1.29683 & -0.34 & 1.281552 & -1.52 & 1.303854 & 0.2 \\
\hline 30 & 1.294449 & 1.291352 & -0.24 & 1.281552 & -1 & 1.296077 & 0.13 \\
\hline 40 & 1.291037 & 1.28876 & -0.18 & 1.281552 & -0.73 & 1.292321 & 0.1 \\
\hline Error range & & & $-1.11 \sim-0.18$ & & $-14.5 \sim-0.73$ & & $0.1 \sim 2.11$ \\
\hline$p$ & & & & 0.95 & & & \\
\hline$n$ & Exact solution & Method 1 & Relative error (\%) & Method 2 & Relative error (\%) & Method 3 & Relative error (\%) \\
\hline 3 & 1.938445 & 1.954945 & 0.85 & - & - & 1.978771 & 2.08 \\
\hline 4 & 1.829525 & 1.83853 & 0.49 & - & - & 1.853356 & 1.3 \\
\hline 5 & 1.77926 & 1.784694 & 0.31 & - & - & 1.796427 & 0.96 \\
\hline 6 & 1.75043 & 1.753874 & 0.20 & - & - & 1.763918 & 0.77 \\
\hline 7 & 1.731816 & 1.734234 & 0.14 & 1.644854 & -5.02 & 1.742891 & 0.64 \\
\hline 8 & 1.718722 & 1.72031 & 0.09 & 1.644854 & -4.3 & 1.728176 & 0.55 \\
\hline 9 & 1.709037 & 1.710337 & 0.08 & 1.644854 & -3.76 & 1.717302 & 0.48 \\
\hline 10 & 1.701662 & 1.702589 & 0.05 & 1.644854 & -3.34 & 1.708939 & 0.43 \\
\hline 20 & 1.671163 & 1.67113 & 0 & 1.644854 & -1.57 & 1.6746 & 0.21 \\
\hline 30 & 1.661982 & 1.661913 & 0 & 1.644854 & -1.03 & 1.664222 & 0.13 \\
\hline 40 & 1.657536 & 1.657417 & 0 & 1.644854 & -0.77 & 1.659212 & 0.1 \\
\hline Error range & & & $0 \sim 0.85$ & & $-5.02 \sim-0.77$ & & $0.1 \sim 2.08$ \\
\hline
\end{tabular}

When $C=0.9, p=0.7 \sim 0.95$, and $n=3 \sim 40$, the absolute values of the average and maximum relative errors of method 1 are $2.28 \%, 12.33 \%$, the absolute values of the average and maximum relative errors of method 2 are $10.33 \%, 104.4 \%$, and the absolute values of the average and maximum relative errors of method 3 are $1.38 \%, 3.9 \%$. It 
TABLE 2: Corollary results of coefficient $k(C=0.6)$.

\begin{tabular}{|c|c|c|c|c|c|c|c|}
\hline$p$ & & & & 0.7 & & & \\
\hline$n$ & Exact solution & Method 1 & Relative error (\%) & Method 2 & Relative error (\%) & Method 3 & Relative error (\%) \\
\hline 3 & 0.799895 & 0.58474 & -26.9 & 0.627209 & -21.6 & 0.801897 & 0.25 \\
\hline 4 & 0.733265 & 0.578577 & -21.1 & - & - & 0.733647 & 0.05 \\
\hline 5 & 0.698054 & 0.577128 & -17.32 & 0.702867 & 0.69 & 0.69811 & 0.01 \\
\hline 6 & 0.675672 & 0.576407 & -14.69 & 0.659712 & -2.36 & 0.675599 & -0.01 \\
\hline 7 & 0.659758 & 0.575744 & -12.73 & 0.642013 & -2.69 & 0.659767 & 0 \\
\hline 8 & 0.647848 & 0.574975 & -11.25 & 0.630945 & -2.61 & 0.647881 & 0.01 \\
\hline 9 & 0.63852 & 0.574221 & -10.07 & 0.622911 & -2.44 & 0.63855 & 0 \\
\hline 10 & 0.630996 & 0.57343 & -9.12 & 0.616635 & -2.28 & 0.630981 & 0 \\
\hline 20 & 0.594181 & 0.566305 & -4.69 & 0.586898 & -1.23 & 0.594198 & 0 \\
\hline 30 & 0.579687 & 0.561364 & -3.16 & 0.574889 & -0.83 & 0.57971 & 0 \\
\hline 40 & 0.571485 & 0.557856 & -2.38 & 0.567906 & -0.63 & 0.571498 & 0 \\
\hline Error range & & & $-26.9 \sim-2.38$ & & $-21.6 \sim 0.69$ & & $0 \sim 0.25$ \\
\hline
\end{tabular}

\begin{tabular}{|c|c|c|c|c|c|c|c|}
\hline$p$ & & & & 0.8 & & & \\
\hline$n$ & Exact solution & Method 1 & Relative error (\%) & Method 2 & Relative error (\%) & Method 3 & Relative error (\%) \\
\hline 3 & 1.211267 & 1.111396 & -8.25 & 0.867739 & -28.36 & 1.216148 & 0.4 \\
\hline 4 & 1.110931 & 1.038738 & -6.5 & - & - & 1.113718 & 0.25 \\
\hline 5 & 1.060245 & 1.003604 & -5.34 & 1.082299 & 2.08 & 1.062497 & 0.21 \\
\hline 6 & 1.02914 & 0.982315 & -4.55 & 1.010636 & -1.8 & 1.030933 & 0.17 \\
\hline 7 & 1.007569 & 0.967799 & -3.95 & 0.983455 & -2.39 & 1.009184 & 0.16 \\
\hline 8 & 0.991641 & 0.957063 & -3.49 & 0.967535 & -2.43 & 0.993113 & 0.15 \\
\hline 9 & 0.97935 & 0.948756 & -3.12 & 0.95652 & -2.33 & 0.980658 & 0.13 \\
\hline 10 & 0.969502 & 0.942029 & -2.83 & 0.948208 & -2.2 & 0.970662 & 0.12 \\
\hline 20 & 0.922891 & 0.909472 & -1.45 & 0.911562 & -1.23 & 0.923535 & 0.07 \\
\hline 30 & 0.905238 & 0.89636 & -0.98 & 0.897651 & -0.84 & 0.905674 & 0.05 \\
\hline 40 & 0.895406 & 0.888792 & -0.74 & 0.889712 & -0.64 & 0.895735 & 0.04 \\
\hline Error range & & & $-8.25 \sim-0.74$ & & $-28.36 \sim 2.08$ & & $0.04 \sim 0.4$ \\
\hline
\end{tabular}

\begin{tabular}{|c|c|c|c|c|c|c|c|}
\hline$p$ & & & & 0.9 & & & \\
\hline$n$ & Exact solution & Method 1 & Relative error (\%) & Method 2 & Relative error (\%) & Method 3 & Relative error (\%) \\
\hline 3 & 1.805399 & 1.77636 & -1.61 & - & - & 1.812208 & 0.38 \\
\hline 4 & 1.6497 & 1.628998 & -1.25 & - & - & 1.655357 & 0.34 \\
\hline 5 & 1.574205 & 1.557654 & -1.05 & 1.618757 & 2.83 & 1.579205 & 0.32 \\
\hline 6 & 1.528963 & 1.514778 & -0.93 & 1.506009 & -1.5 & 1.533232 & 0.28 \\
\hline 7 & 1.498267 & 1.485975 & -0.82 & 1.464439 & -2.26 & 1.502045 & 0.25 \\
\hline 8 & 1.475927 & 1.465039 & -0.74 & 1.440912 & -2.37 & 1.479285 & 0.23 \\
\hline 9 & 1.458642 & 1.449051 & -0.66 & 1.42511 & -2.3 & 1.461825 & 0.22 \\
\hline 10 & 1.445112 & 1.436289 & -0.61 & 1.413474 & -2.19 & 1.447934 & 0.2 \\
\hline 20 & 1.382494 & 1.377987 & -0.33 & 1.365121 & -1.26 & 1.384065 & 0.11 \\
\hline 30 & 1.359598 & 1.356631 & -0.22 & 1.347844 & -0.86 & 1.360658 & 0.08 \\
\hline 40 & 1.347075 & 1.344737 & -0.17 & 1.338184 & -0.66 & 1.347853 & 0.06 \\
\hline Error range & & & $-1.61 \sim-0.17$ & & $-2.37 \sim 2.83$ & & $0.06 \sim 0.38$ \\
\hline$p$ & & & & 0.95 & & & \\
\hline$n$ & Exact solution & Method 1 & Relative error (\%) & Method 2 & Relative error (\%) & Method 3 & Relative error (\%) \\
\hline 3 & 2.30676 & 2.308331 & 0.07 & - & - & 2.312866 & 0.26 \\
\hline 4 & 2.102118 & 2.104901 & 0.13 & - & - & 2.108769 & 0.32 \\
\hline 5 & 2.004726 & 2.006578 & 0.09 & 2.065685 & 3.04 & 2.010919 & 0.31 \\
\hline 6 & 1.946573 & 1.948 & 0.07 & 1.918951 & -1.42 & 1.952369 & 0.3 \\
\hline 7 & 1.90781 & 1.9086 & 0.04 & 1.86521 & -2.23 & 1.912919 & 0.27 \\
\hline 8 & 1.879692 & 1.880188 & 0.03 & 1.835126 & -2.37 & 1.884289 & 0.24 \\
\hline 9 & 1.858126 & 1.85848 & 0.02 & 1.815139 & -2.31 & 1.862426 & 0.23 \\
\hline 10 & 1.841239 & 1.841258 & 0 & 1.800564 & -2.21 & 1.8451 & 0.21 \\
\hline 20 & 1.764225 & 1.764128 & -0.01 & 1.741567 & -1.28 & 1.766358 & 0.12 \\
\hline 30 & 1.736488 & 1.736406 & 0 & 1.721125 & -0.88 & 1.737963 & 0.08 \\
\hline 40 & 1.721437 & 1.721317 & -0.01 & 1.709825 & -0.67 & 1.72256 & 0.07 \\
\hline Error range & & & $-0.01 \sim 0.07$ & & $-2.37 \sim 3.04$ & & $0.07 \sim 0.26$ \\
\hline
\end{tabular}

can be seen that the precision of method 2 is the lowest, method 1 is in the middle, and method 3 has the highest precision.
With an absolute value of relative error not exceeding $5 \%$, the applicable range of the method 1 is $n>10$, and the applicable range of method 2 is $n>7$, and method 3 satisfies 
TABLE 3: Corollary results of coefficient $k(C=0.7)$.

\begin{tabular}{|c|c|c|c|c|c|c|c|}
\hline$p$ & & & & 0.7 & & & \\
\hline$n$ & Exact solution & Method 1 & Relative error (\%) & Method 2 & Relative error (\%) & Method 3 & Relative error (\%) \\
\hline 3 & 1.056856 & 0.823382 & -22.09 & - & - & 1.041203 & -1.48 \\
\hline 4 & 0.92804 & 0.770563 & -16.97 & 0.982941 & 5.92 & 0.919138 & -0.96 \\
\hline 5 & 0.861097 & 0.742317 & -13.79 & 0.850421 & -1.24 & 0.855142 & -0.69 \\
\hline 6 & 0.818649 & 0.723444 & -11.63 & 0.799625 & -2.32 & 0.814274 & -0.53 \\
\hline 7 & 0.788632 & 0.709445 & -10.04 & 0.769229 & -2.46 & 0.785322 & -0.42 \\
\hline 8 & 0.766156 & 0.698382 & -8.85 & 0.747888 & -2.38 & 0.763447 & -0.35 \\
\hline 9 & 0.748413 & 0.689293 & -7.9 & 0.731641 & -2.24 & 0.74618 & -0.3 \\
\hline 10 & 0.73401 & 0.681582 & -7.14 & 0.718652 & -2.09 & 0.732107 & -0.26 \\
\hline 20 & 0.663438 & 0.639206 & -3.65 & 0.655701 & -1.17 & 0.662707 & -0.11 \\
\hline 30 & 0.635261 & 0.619653 & -2.46 & 0.6302 & -0.8 & 0.634827 & -0.07 \\
\hline 40 & 0.619144 & 0.607677 & -1.85 & 0.615421 & -0.6 & 0.61886 & -0.05 \\
\hline Error range & & & $-22.09 \sim-1.85$ & & $-2.46 \sim 5.92$ & & $-1.48 \sim-0.05$ \\
\hline$p$ & & & & 0.8 & & & \\
\hline$n$ & Exact solution & Method 1 & Relative error (\%) & Method 2 & Relative error (\%) & Method 3 & Relative error (\%) \\
\hline 3 & 1.528369 & 1.412346 & -7.59 & - & - & 1.507871 & -1.34 \\
\hline 4 & 1.343264 & 1.267934 & -5.61 & 1.458218 & 8.56 & 1.333216 & -0.75 \\
\hline 5 & 1.251379 & 1.1953 & -4.48 & 1.248493 & -0.23 & 1.245424 & -0.48 \\
\hline 6 & 1.19489 & 1.150102 & -3.75 & 1.173717 & -1.77 & 1.190894 & -0.33 \\
\hline 7 & 1.15592 & 1.118601 & -3.23 & 1.131398 & -2.12 & 1.153027 & -0.25 \\
\hline 8 & 1.1271 & 1.09512 & -2.84 & 1.102841 & -2.15 & 1.12485 & -0.2 \\
\hline 9 & 1.104595 & 1.076739 & -2.52 & 1.081717 & -2.07 & 1.102872 & -0.16 \\
\hline 10 & 1.0865 & 1.061783 & -2.27 & 1.06519 & -1.96 & 1.085136 & -0.13 \\
\hline 20 & 1.00039 & 0.988779 & -1.16 & 0.988889 & -1.15 & 0.999985 & -0.04 \\
\hline 30 & 0.967035 & 0.959519 & -0.78 & 0.959368 & -0.79 & 0.966858 & -0.02 \\
\hline 40 & 0.948261 & 0.942748 & -0.58 & 0.942525 & -0.6 & 0.948161 & -0.01 \\
\hline Error range & & & $-7.59 \sim-0.58$ & & $-2.15 \sim 8.56$ & & $-1.34 \sim-0.01$ \\
\hline$p$ & & & & 0.9 & & & \\
\hline$n$ & Exact solution & Method 1 & Relative error (\%) & Method 2 & Relative error (\%) & Method 3 & Relative error (\%) \\
\hline 3 & 2.219999 & 2.162173 & -2.6 & - & - & 2.190339 & -1.34 \\
\hline 4 & 1.943907 & 1.912762 & -1.6 & 2.14235 & 10.21 & 1.931962 & -0.61 \\
\hline 5 & 1.81224 & 1.790519 & -1.2 & 1.819984 & 0.43 & 1.806181 & -0.33 \\
\hline 6 & 1.733163 & 1.716398 & -0.97 & 1.708671 & -1.41 & 1.729754 & -0.2 \\
\hline 7 & 1.679532 & 1.665911 & -0.81 & 1.647691 & -1.9 & 1.67754 & -0.12 \\
\hline 8 & 1.640374 & 1.628914 & -0.7 & 1.60763 & -2 & 1.63918 & -0.07 \\
\hline 9 & 1.610364 & 1.600302 & -0.62 & 1.578625 & -1.97 & 1.609567 & -0.05 \\
\hline 10 & 1.586351 & 1.577401 & -0.56 & 1.556317 & -1.89 & 1.585873 & -0.03 \\
\hline 20 & 1.474504 & 1.470441 & -0.28 & 1.457608 & -1.15 & 1.474811 & 0.02 \\
\hline 30 & 1.432615 & 1.429924 & -0.19 & 1.421102 & -0.8 & 1.432884 & 0.02 \\
\hline 40 & 1.409306 & 1.407292 & -0.14 & 1.400619 & -0.62 & 1.409556 & 0.02 \\
\hline Error range & & & $-2.6 \sim-0.14$ & & $-2 \sim 10.21$ & & $-1.34 \sim 0.02$ \\
\hline$p$ & & & & 0.95 & & & \\
\hline$n$ & Exact solution & Method 1 & Relative error (\%) & Method 2 & Relative error (\%) & Method 3 & Relative error (\%) \\
\hline 3 & 2.8089821 & 2.769737 & -1.4 & - & - & 2.769095 & -1.42 \\
\hline 4 & 2.4530226 & 2.438936 & -0.57 & 2.717347 & 10.8 & 2.437778 & -0.62 \\
\hline 5 & 2.2858326 & 2.278508 & -0.32 & 2.300963 & 0.66 & 2.278716 & -0.31 \\
\hline 6 & 2.186787 & 2.181884 & -0.22 & 2.158486 & -1.29 & 2.183009 & -0.17 \\
\hline 7 & 2.120093 & 2.116571 & -0.17 & 2.081324 & -1.83 & 2.118108 & -0.09 \\
\hline 8 & 2.071657 & 2.069042 & -0.13 & 2.031161 & -1.95 & 2.070709 & -0.05 \\
\hline 9 & 2.034752 & 2.032585 & -0.11 & 1.995165 & -1.95 & 2.034297 & -0.02 \\
\hline 10 & 2.0052348 & 2.003434 & -0.09 & 1.967689 & -1.87 & 2.00528 & 0 \\
\hline 20 & 1.8702006 & 1.869459 & -0.04 & 1.848538 & -1.16 & 1.870855 & 0.03 \\
\hline 30 & 1.820288 & 1.819858 & -0.02 & 1.805492 & -0.81 & 1.820876 & 0.03 \\
\hline 40 & 1.7927441 & 1.7925 & -0.01 & 1.781563 & -0.62 & 1.793272 & 0.03 \\
\hline Error range & & & $-1.4 \sim-0.01$ & & $-1.95 \sim 10.8$ & & $-1.42 \sim 0.03$ \\
\hline
\end{tabular}

all the values of $n$ in the above study. When $n<5$, the difference of the relative error calculated by method 2 is the largest, indicating that method 2 is more suitable for the approximate solution of the coefficient $k$ when $n \geq 5$. It can be seen from the figure that when $p$ is increased from 0.7 to 0.95 , the absolute value of relative error calculated by these 
TABLE 4: Corollary results of coefficient $k(C=0.8)$.

\begin{tabular}{|c|c|c|c|c|c|c|c|}
\hline$p$ & & & & 0.7 & & & \\
\hline$n$ & Exact solution & Method 1 & Relative error (\%) & Method 2 & Relative error (\%) & Method 3 & Relative error (\%) \\
\hline 3 & 1.441459 & 1.198475 & -16.86 & 2.240555 & 55.44 & 1.392952 & -3.37 \\
\hline 4 & 1.199003 & 1.056408 & -11.89 & 1.233037 & 2.84 & 1.171917 & -2.26 \\
\hline 5 & 1.079699 & 0.980532 & -9.18 & 1.065791 & -1.29 & 1.06182 & -1.66 \\
\hline 6 & 1.006262 & 0.931047 & -7.47 & 0.984826 & -2.13 & 0.993137 & -1.3 \\
\hline 7 & 0.955358 & 0.895292 & -6.29 & 0.933649 & -2.27 & 0.945115 & -1.07 \\
\hline 8 & 0.917479 & 0.867706 & -5.42 & 0.897132 & -2.22 & 0.909135 & -0.91 \\
\hline 9 & 0.887813 & 0.845589 & -4.76 & 0.869215 & -2.09 & 0.880892 & -0.78 \\
\hline 10 & 0.863964 & 0.827366 & -4.24 & 0.846899 & -1.98 & 0.857968 & -0.69 \\
\hline 20 & 0.748168 & 0.733359 & -1.98 & 0.739788 & -1.12 & 0.745878 & -0.31 \\
\hline 30 & 0.70254 & 0.693546 & -1.28 & 0.697122 & -0.77 & 0.70116 & -0.2 \\
\hline 40 & 0.676569 & 0.670218 & -0.94 & 0.672596 & -0.59 & 0.675594 & -0.14 \\
\hline Error range & & & $-16.86 \sim-0.94$ & & $-2.27 \sim 55.44$ & & $-3.37 \sim-0.14$ \\
\hline$p$ & & & & 0.8 & & & \\
\hline$n$ & Exact solution & Method 1 & Relative error (\%) & Method 2 & Relative error (\%) & Method 3 & Relative error (\%) \\
\hline 3 & 2.015963 & 1.864198 & -7.53 & 3.350253 & 66.19 & 1.959245 & -2.81 \\
\hline 4 & 1.67476 & 1.595514 & -4.73 & 1.756294 & 4.87 & 1.644344 & -1.82 \\
\hline 5 & 1.513834 & 1.461578 & -3.45 & 1.508846 & -0.33 & 1.494208 & -1.3 \\
\hline 6 & 1.417233 & 1.378831 & -2.71 & 1.395374 & -1.54 & 1.403193 & -0.99 \\
\hline 7 & 1.35164 & 1.321513 & -2.23 & 1.326381 & -1.87 & 1.340847 & -0.8 \\
\hline 8 & 1.303523 & 1.278875 & -1.89 & 1.278522 & -1.92 & 1.294852 & -0.67 \\
\hline 9 & 1.266389 & 1.245617 & -1.64 & 1.242699 & -1.87 & 1.259186 & -0.57 \\
\hline 10 & 1.236572 & 1.218831 & -1.43 & 1.214529 & -1.78 & 1.230522 & -0.49 \\
\hline 20 & 1.096332 & 1.089234 & -0.65 & 1.084534 & -1.08 & 1.094046 & -0.21 \\
\hline 30 & 1.042614 & 1.038382 & -0.41 & 1.03481 & -0.75 & 1.041283 & -0.13 \\
\hline 40 & 1.012475 & 1.009494 & -0.29 & 1.00666 & -0.57 & 1.011538 & -0.09 \\
\hline Error range & & & $-7.53 \sim-0.29$ & & $-1.92 \sim 66.19$ & & $-2.81 \sim-0.09$ \\
\hline$p$ & & & & 0.9 & & & \\
\hline$n$ & Exact solution & Method 1 & Relative error (\%) & Method 2 & Relative error (\%) & Method 3 & Relative error (\%) \\
\hline 3 & 2.870411 & 2.734096 & -4.75 & 4.95068 & 72.47 & 2.800163 & -2.45 \\
\hline 4 & 2.372481 & 2.313091 & -2.5 & 2.520845 & 6.25 & 2.337578 & -1.47 \\
\hline 5 & 2.145211 & 2.11023 & -1.63 & 2.153216 & 0.37 & 2.124036 & -0.99 \\
\hline 6 & 2.011888 & 1.988188 & -1.18 & 1.989734 & -1.1 & 1.997447 & -0.72 \\
\hline 7 & 1.922926 & 1.905291 & -0.92 & 1.892856 & -1.56 & 1.912164 & -0.56 \\
\hline 8 & 1.858496 & 1.844579 & -0.75 & 1.827017 & -1.69 & 1.850058 & -0.45 \\
\hline 9 & 1.809182 & 1.797828 & -0.63 & 1.778539 & -1.69 & 1.802401 & -0.37 \\
\hline 10 & 1.770182 & 1.760544 & -0.54 & 1.740922 & -1.65 & 1.764428 & -0.33 \\
\hline 20 & 1.58978 & 1.586399 & -0.21 & 1.573196 & -1.04 & 1.587908 & -0.12 \\
\hline 30 & 1.522643 & 1.520797 & -0.12 & 1.511473 & -0.73 & 1.521655 & -0.06 \\
\hline 40 & 1.485504 & 1.484164 & -0.09 & 1.477065 & -0.57 & 1.484813 & -0.05 \\
\hline Error range & & & $-4.75 \sim-0.09$ & & $-1.69 \sim 72.47$ & & $-2.45 \sim-0.05$ \\
\hline$p$ & & & & 0.95 & & & \\
\hline$n$ & Exact solution & Method 1 & Relative error (\%) & Method 2 & Relative error (\%) & Method 3 & Relative error (\%) \\
\hline 3 & 3.603836 & 3.451824 & -4.22 & 6.293786 & 74.64 & 3.519022 & -2.35 \\
\hline 4 & 2.968097 & 2.908834 & -2 & 3.169819 & 6.8 & 2.928243 & -1.34 \\
\hline 5 & 2.682675 & 2.65053 & -1.2 & 2.700191 & 0.65 & 2.659239 & -0.87 \\
\hline 6 & 2.5169789 & 2.496323 & -0.82 & 2.493566 & -0.93 & 2.501342 & -0.62 \\
\hline 7 & 2.4069464 & 2.392608 & -0.6 & 2.372342 & -1.44 & 2.395769 & -0.46 \\
\hline 8 & 2.3278784 & 2.31704 & -0.47 & 2.29066 & -1.6 & 2.319351 & -0.37 \\
\hline 9 & 2.2677779 & 2.259295 & -0.37 & 2.230948 & -1.62 & 2.261001 & -0.3 \\
\hline 10 & 2.220237 & 2.213298 & -0.31 & 2.184893 & -1.59 & 2.214701 & -0.25 \\
\hline 20 & 2.003516 & 2.001622 & -0.09 & 1.982907 & -1.03 & 2.001987 & -0.08 \\
\hline 30 & 1.924118 & 1.92322 & -0.05 & 1.910046 & -0.73 & 1.923348 & -0.04 \\
\hline 40 & 1.880421 & 1.879741 & -0.04 & 1.869768 & -0.57 & 1.879932 & -0.03 \\
\hline Error range & & & $-4.22 \sim-0.04$ & & $-1.62 \sim 74.64$ & & $-2.35 \sim-0.03$ \\
\hline
\end{tabular}


TABLE 5: Corollary results of coefficient $k(C=0.9)$.

\begin{tabular}{|c|c|c|c|c|c|c|c|}
\hline$p$ & & & & 0.7 & & & \\
\hline$n$ & Exact solution & Method 1 & Relative error (\%) & Method 2 & Relative error (\%) & Method 3 & Relative error (\%) \\
\hline 3 & 2.227727 & 1.95304 & -12.33 & 3.927345 & 76.29 & 2.172989 & -2.46 \\
\hline 4 & 1.692664 & 1.592182 & -5.94 & 1.818662 & 7.44 & 1.627362 & -3.86 \\
\hline 5 & 1.455446 & 1.409432 & -3.16 & 1.468263 & 0.88 & 1.406016 & -3.4 \\
\hline 6 & 1.317233 & 1.294781 & -1.7 & 1.30651 & -0.81 & 1.279076 & -2.9 \\
\hline 7 & 1.225071 & 1.214403 & -0.87 & 1.208123 & -1.38 & 1.19427 & -2.51 \\
\hline 8 & 1.15805 & 1.154134 & -0.34 & 1.139942 & -1.56 & 1.132505 & -2.21 \\
\hline 9 & 1.106482 & 1.106785 & 0.03 & 1.088974 & -1.58 & 1.084946 & -1.95 \\
\hline 10 & 1.065516 & 1.068362 & 0.27 & 1.048945 & -1.56 & 1.046877 & -1.75 \\
\hline 20 & 0.873674 & 0.88102 & 0.84 & 0.86496 & -1 & 0.866203 & -0.86 \\
\hline 30 & 0.800592 & 0.806951 & 0.79 & 0.794931 & -0.71 & 0.79604 & -0.57 \\
\hline 40 & 0.759473 & 0.764901 & 0.71 & 0.755368 & -0.54 & 0.756278 & -0.42 \\
\hline Error range & & & $-12.33 \sim 0.84$ & & $-1.58 \sim 76.29$ & & $-3.86 \sim-0.42$ \\
\hline$p$ & & & & 0.8 & & & \\
\hline$n$ & Exact solution & Method 1 & Relative error (\%) & Method 2 & Relative error (\%) & Method 3 & Relative error (\%) \\
\hline 3 & 3.039117 & 2.738367 & -9.9 & 5.814814 & 91.33 & 3.03893 & -0.01 \\
\hline 4 & 2.294540 & 2.186456 & -4.71 & 2.534914 & 10.48 & 2.236538 & -2.53 \\
\hline 5 & 1.975691 & 1.92394 & -2.62 & 2.023345 & 2.41 & 1.927019 & -2.46 \\
\hline 6 & 1.794452 & 1.766061 & -1.58 & 1.797266 & 0.16 & 1.755281 & -2.18 \\
\hline 7 & 1.675329 & 1.658734 & -0.99 & 1.664035 & -0.67 & 1.643227 & -1.92 \\
\hline 8 & 1.589881 & 1.580105 & -0.61 & 1.573872 & -1.01 & 1.563058 & -1.69 \\
\hline 9 & 1.525238 & 1.519469 & -0.38 & 1.507702 & -1.15 & 1.502188 & -1.51 \\
\hline 10 & 1.474096 & 1.470974 & -0.21 & 1.456488 & -1.19 & 1.454012 & -1.36 \\
\hline 20 & 1.240591 & 1.243243 & 0.21 & 1.229623 & -0.88 & 1.232126 & -0.68 \\
\hline 30 & 1.154152 & 1.15689 & 0.24 & 1.146775 & -0.64 & 1.148946 & -0.45 \\
\hline 40 & 1.106253 & 1.108681 & 0.22 & 1.100748 & -0.5 & 1.102528 & -0.34 \\
\hline Error range & & & $-9.9 \sim 0.24$ & & $-1.19 \sim 91.33$ & & $-2.53 \sim-0.01$ \\
\hline$p$ & & & & 0.9 & & & \\
\hline$n$ & Exact solution & Method 1 & Relative error (\%) & Method 2 & Relative error (\%) & Method 3 & Relative error (\%) \\
\hline 3 & 4.258037 & 3.827763 & -10.11 & 8.552616 & 101.86 & 4.340567 & 1.94 \\
\hline 4 & 3.187642 & 3.026395 & -5.06 & 3.594739 & 12.77 & 3.144705 & -1.35 \\
\hline 5 & 2.742235 & 2.658306 & -3.06 & 2.842316 & 3.65 & 2.698114 & -1.61 \\
\hline 6 & 2.493491 & 2.442339 & -2.05 & 2.518136 & 0.99 & 2.456109 & -1.5 \\
\hline 7 & 2.332315 & 2.298158 & -1.46 & 2.331059 & -0.05 & 2.301023 & -1.34 \\
\hline 8 & 2.218358 & 2.193841 & -1.11 & 2.206595 & -0.53 & 2.191628 & -1.2 \\
\hline 9 & 2.132705 & 2.114345 & -0.86 & 2.116515 & -0.76 & 2.109516 & -1.09 \\
\hline 10 & 2.065574 & 2.051333 & -0.69 & 2.047597 & -0.87 & 2.045142 & -0.99 \\
\hline 20 & 1.765161 & 1.762608 & -0.14 & 1.751624 & -0.77 & 1.756306 & -0.5 \\
\hline 30 & 1.656991 & 1.656266 & -0.04 & 1.647536 & -0.57 & 1.651498 & -0.33 \\
\hline 40 & 1.597842 & 1.597666 & -0.01 & 1.590631 & -0.45 & 1.593871 & -0.25 \\
\hline Error range & & & $-10.11 \sim-0.01$ & & $-0.87 \sim 101.86$ & & $-1.61 \sim 1.94$ \\
\hline$p$ & & & & 0.95 & & & \\
\hline$n$ & Exact solution & Method 1 & Relative error (\%) & Method 2 & Relative error (\%) & Method 3 & Relative error (\%) \\
\hline 3 & 5.311158 & 4.75345 & -10.5 & 10.85606 & 104.4 & 5.457418 & 2.75 \\
\hline 4 & 3.955887 & 3.743654 & -5.36 & 4.5002 & 13.76 & 3.924381 & -0.8 \\
\hline 5 & 3.399631 & 3.286259 & -3.33 & 3.543087 & 4.22 & 3.35901 & -1.19 \\
\hline 6 & 3.0916004 & 3.020273 & -2.31 & 3.134426 & 1.39 & 3.055638 & -1.16 \\
\hline 7 & 2.8934486 & 2.844041 & -1.71 & 2.90053 & 0.24 & 2.862743 & -1.06 \\
\hline 8 & 2.7540745 & 2.717418 & -1.33 & 2.746018 & -0.29 & 2.727541 & -0.96 \\
\hline 9 & 2.6498059 & 2.62142 & -1.07 & 2.634866 & -0.56 & 2.626593 & -0.88 \\
\hline 10 & 2.568089 & 2.545597 & -0.88 & 2.550266 & -0.69 & 2.547805 & -0.79 \\
\hline 20 & 2.207616 & 2.201785 & -0.26 & 2.19221 & -0.7 & 2.19873 & -0.4 \\
\hline 30 & 2.079723 & 2.077018 & -0.13 & 2.06865 & -0.53 & 2.074132 & -0.27 \\
\hline 40 & 2.010191 & 2.008534 & -0.08 & 2.00167 & -0.42 & 2.006152 & -0.2 \\
\hline Error range & & & $-10.5 \sim-0.08$ & & $-0.7 \sim 104.4$ & & $-1.19 \sim 2.75$ \\
\hline
\end{tabular}




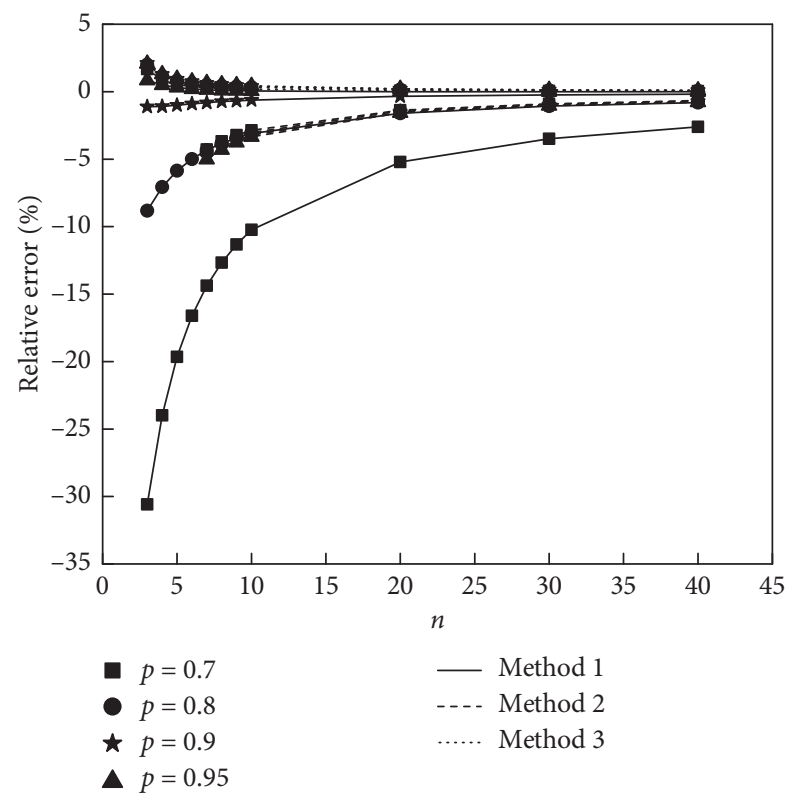

FIgURE 1: Relative error $(C=0.5)$.

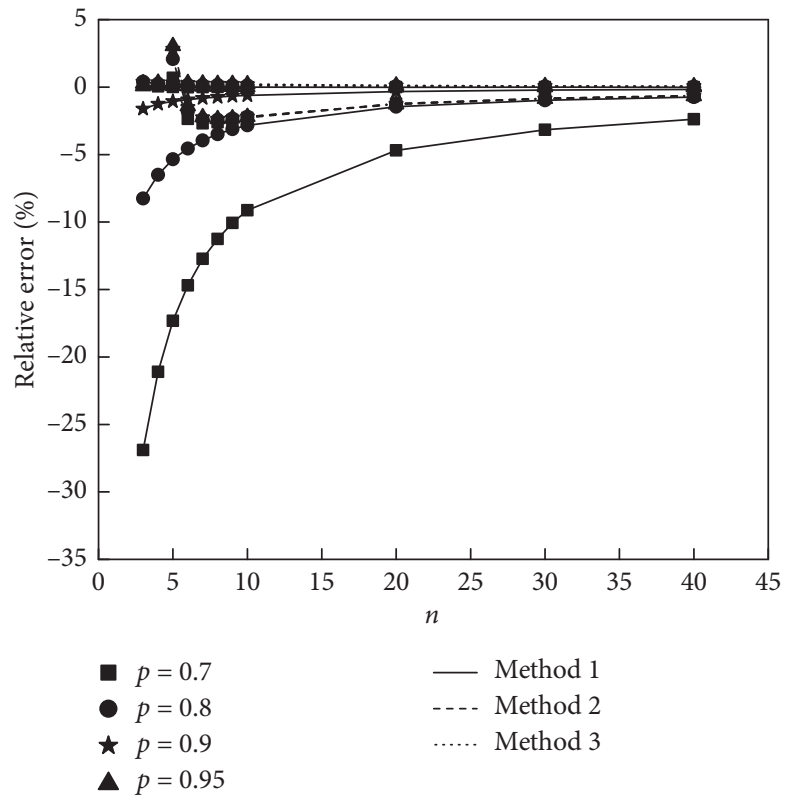

Figure 2: Relative error $(C=0.6)$.

three methods has a decreasing trend, indicating that the precision of these three methods is improved with the increase of $p$. With the increase of $n$, the absolute value of the relative error calculated by these three methods has a decreasing trend, indicating that the precision of these three methods is improved with the increase of $n$. When $n>10$, the relative error trend curve of the coefficient $k$ calculated by these three methods tends to be gentle, indicating that the accuracy of these three methods is improved with the increase of $n$. And the relative error calculated by method 3 


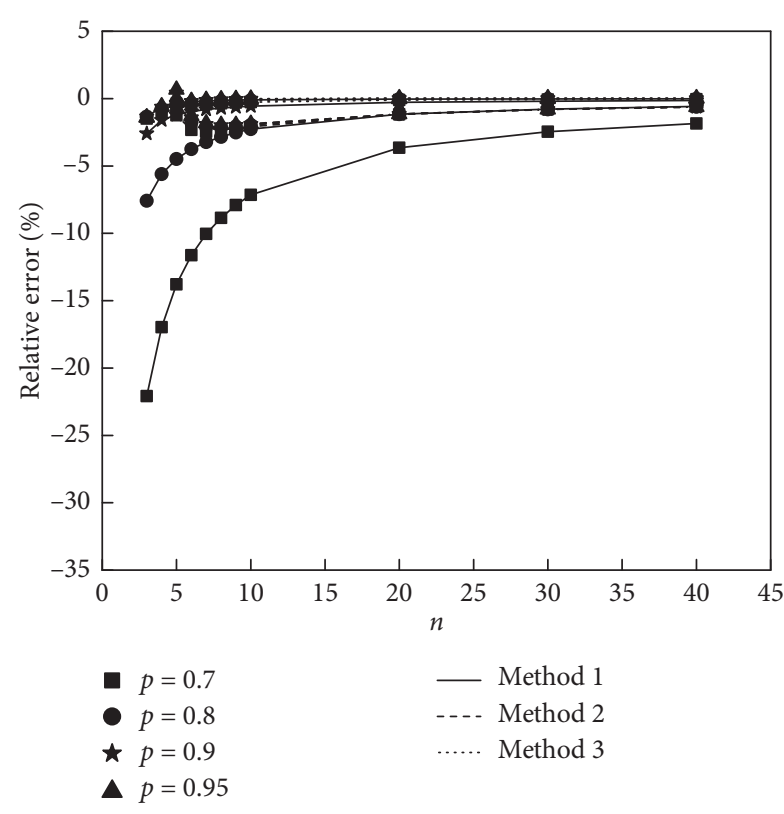

Figure 3: Relative error $(C=0.7)$.

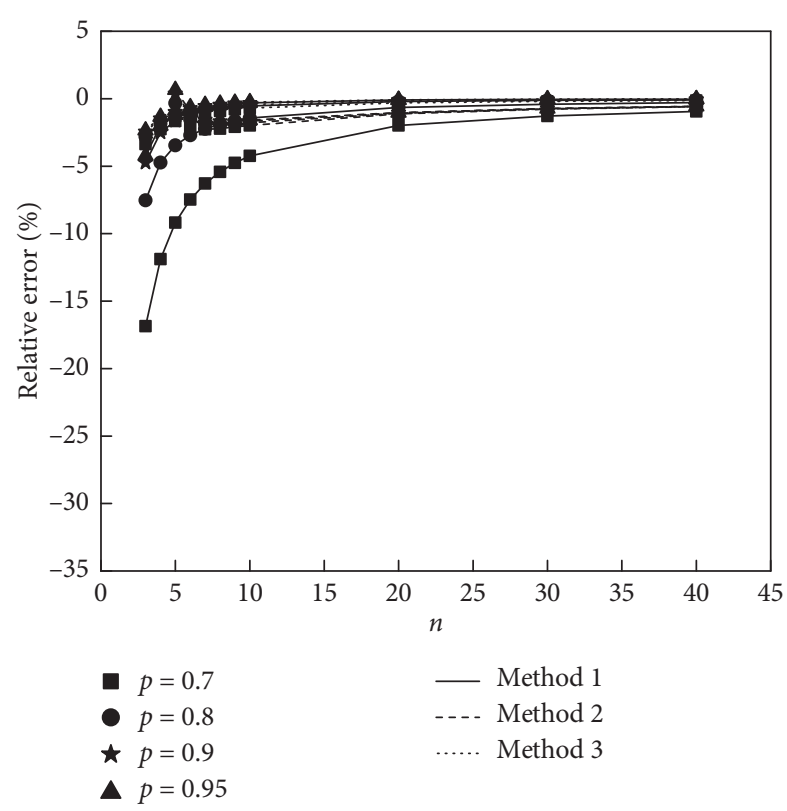

Figure 4: Relative error $(C=0.8)$.

fluctuates around 0 , and there is no large variability. In summary, method 3 has a wider range of applications than the first two methods.

\section{Concluding Remarks}

Through the study of the inference method of the noncentral $\mathrm{t}$-distribution value, the following main conclusions can be obtained:

(1) By comparing and analyzing the error range of coefficient $k$ under different conditions, it is shown that the quantile value corollary method proposed in

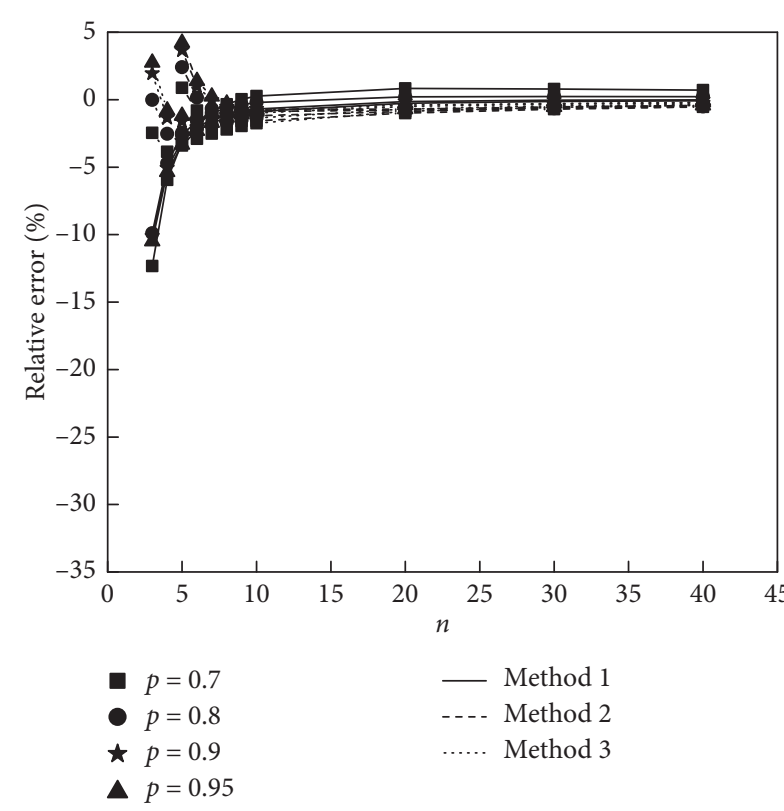

Figure 5: Relative error $(C=0.9)$.

this paper is more accurate than the current approximation method.

(2) By comparing the influence of different number of samples $n$ on the coefficient $k$, it indicates that the proposed method in this paper has a wider scope of application.

(3) The method of inferring the quantile value proposed in this paper does not need to repeatedly check the value table and does not need to perform linear interpolation calculation, which simplifies the calculation process and has better convenience.

\section{Data Availability}

Some or all data, models, or codes that support the findings of this study are available from the corresponding author upon reasonable request.

\section{Conflicts of Interest}

The authors declare that they have no conflicts of interest.

\section{Authors' Contributions}

Jun Gao and Jitao Yao contributed the central idea, and Jun Gao analysed most of the data and wrote the initial draft of the paper. The remaining authors contributed to refining the ideas, carrying out additional analyses, and finalizing this paper.

\section{Acknowledgments}

The authors would like to gratefully acknowledge the financial support from the National Natural Science Foundations of China (51278401). 


\section{References}

[1] D. Meng, T. Xie, P. Wu, S.-P. Zhu, Z. Hu, and Y. Li, "Uncertainty-based design and optimization using first order saddle point approximation method for multidisciplinary engineering systems," ASCE-ASME Journal of Risk and Uncertainty in Engineering Systems, Part A: Civil Engineering, vol. 6, no. 3, Article ID 04020028, 2020.

[2] D. Meng, Z. Hu, P. Wu et al., "Reliability-based optimisation for offshore structures using saddlepoint approximation," Proceedings of the Institution of Civil Engineers-Maritime Engineering, vol. 173, no. 2, pp. 33-42, 2020.

[3] H. Li, R. Yuan, and J. Fu, "A reliability modeling for multicomponent systems considering random shocks and multi-state degradation," IEEE Access, vol. 7, Article ID 168805, 2019.

[4] R. Yuan, H. Li, and Q. Wang, "Simulation-based design and optimization and fatigue characteristics for high-speed backplane connector," Advances in Mechanical Engineering, vol. 11, no. 6, pp. 1-10, 2019.

[5] R. Yuan, M. Tang, H. Wang, and H. Li, "A reliability analysis method of accelerated performance degradation based on bayesian strategy," IEEE Access, vol. 7, Article ID 169047, 2019.

[6] Z. Gairong, Probability Theory and Mathematical Statistics, China Commercial Publishing House Press, Beijing, China, 2006.

[7] P. F. Rust, Noncentral T Distribution: Introduction. Wiley StatsRef: Statistics Reference Online, John Wiley \& Sons, Ltd, New Yark, NY, USA, 2014.

[8] A. Harvey and R.-J. Lange, "Volatility modeling with a generalized t distribution," Journal of Time Series Analysis, vol. 38, no. 2, pp. 175-190, 2017.

[9] Y. Fatma Zehra Doğru, "Murat bulut,olcay arslan. doubly reweighted estimators for the parameters of the multivariate t-distribution," Communications in Statistics-Theory and Methods, vol. 47, pp. 4751-4771, 2018.

[10] J. Kim and A. J. Hayter, "Efficient confidence interval methodologies for the non centrality parameter of a non central $t$ distribution," Communications in Statistics-Simulation and Computation, vol. 37, no. 4, pp. 660-678, 2008.

[11] M. Yuan, J. Z. Huang, and Huang, "Regularized parameter estimation of high dimensional t distribution," Journal of Statistical Planning and Inference, vol. 139, no. 7, pp. 22842292, 2009.

[12] C. Huirong, "On an approximate computational method for fractile of non-central t distribution," Journal of Agricultural Sciences, vol. 4, pp. 65-67, 2000.

[13] Y. Jitao and X. Yaokui, "Statistical inference for coefficient of variation in reliability assessment of existing structure," Journal of Building Structure, vol. 31, pp. 101-105, 2010.

[14] Y. Jitao, Reliability Assessment of Existing Structures Based on Uncertainty Reasoning, Science Press, Beijing, China, 2011.

[15] M. Shisong, W. Jinglong, S. Dinghua et al., Statistics Handbook, Science press, Beijing, China, 2003. 